\title{
Site-selective ionization and relaxation dynamics in heterogeneous nanosystems
}

\author{
M. Hoener, ${ }^{1,2, *}$ D. Rolles, ${ }^{1,3}$ A. Aguilar, ${ }^{2}$ R. C. Bilodeau, ${ }^{1,2}$ D. Esteves,,${ }^{2,4}$ P. Olalde Velasco, ${ }^{2,5}$ \\ Z. D. Pešić, ${ }^{6}$ E. Red, ${ }^{2}$ and N. Berrah ${ }^{1}$ \\ ${ }^{1}$ Western Michigan University, Kalamazoo, Michigan, 49008, USA \\ ${ }^{2}$ Lawrence Berkeley National Laboratory, Advanced Light Source, Berkeley, California 94720, USA \\ ${ }^{3}$ Max Planck Advanced Study Group at CFEL, 22761 Hamburg \\ ${ }^{4}$ University of Nevada, Reno, Nevada, USA \\ ${ }^{5}$ Instituto de Ciencias Nucleares, UNAM, Mexico Distrito Federal, 04510, Mexico \\ ${ }^{6}$ Laboratory for Atomic Collision Processes, Institute of Physics, Belgrade, Serbia \\ (Received 9 September 2009; published 1 February 2010)
}

\begin{abstract}
We investigated energy and charge transfer mechanisms as well as fragmentation dynamics in site-selectively ionized heterogeneous core-shell clusters using a high-resolution photoelectron-ion coincidence technique. We show that after inner-shell photoionization, energy or charge is transferred to neighboring atoms and that the subsequent charge localization depends on the site of ionization. Cluster bulk ionization leads to more distinct fragmentation channels than surface ionization. We attribute this to different electronic decay, charge localization, and fragmentation times and conclude that charge transfer and fragmentation dynamics are strongly influenced by the environment of the initially ionized atom.
\end{abstract}

DOI: 10.1103/PhysRevA.81.021201

PACS number(s): 36.40.-c, 73.40.-c, 82.33.Fg

Understanding charge migration and charge transfer processes in nano-scale systems at the atomic level is of utmost importance for a detailed fundamental physical knowledge and for the design of nano devices based on quantum dot structures, nanotubes, or 2D graphene sheets [1,2]. In recent years, advanced many-particle, momentum-resolving electron-ion coincidence techniques have successfully been applied to a variety of small molecular systems in the gas phase to study charge transfer and atomic rearrangement after photoionization [3-5]. Here we report on the application of such a coincidence method to study charge transfer processes in nanoscale objects consisting of several hundred or thousand rare-gas atoms. Touted as an easy-to-produce model system for other nano objects with more direct technological relevance, such rare-gas clusters have been studied extensively by means of electron, ion, and fluorescence spectroscopy. However, when applied individually, all of these techniques can only observe a single channel of the cluster's response to the ionization event. For large systems consisting of several hundred or even thousand atoms, the response of the cluster is too complex and can be understood only by a coincident measurement of several decay products.

Energy and charge transfer processes such as, for example, the interatomic or molecular Coulombic decay (ICD) have been studied extensively, both theoretically and experimentally [3,6-8]. However, almost all of these investigations were focused on molecules $\left(\mathrm{Ne}_{2}, \mathrm{Ar}_{2}, \mathrm{ArKr}\right)$ but not on complex systems like clusters. Averbukh et al. [8] showed that for weakly bound van der Waals clusters, the electron orbital overlap plays a crucial role for the ICD efficiency. An enhancement of the decay rate of two to three orders of magnitude was predicted at the equilibrium geometry. This makes the ICD in clusters even more dramatic than in small molecules and can lead to a preferred transfer direction between different compounds in a mixed system due to

\footnotetext{
*Corresponding author: MHoener@lbl.gov
}

the different polarization forces. Furthermore, the lifetime of the ICD in clusters is shorter for bulk atoms than for surface atoms due to the fact that they have more nearest neighbors than surface atoms [9]. For heterogeneous NeAr dimers, Zobeley et al. [10] calculated very recently that competing electronic decay channels exist. After photoionization of $\mathrm{Ne}$ $2 p$, the system relaxes through (1) the ICD process, where energy is transferred by a virtual photon from the Ne to the Ar atom or (2) the electron transfer mediated decay (ETMD), where an electron is transferred. The latter decay channel of enhanced charge transfer ends up in a neutral Ne state and a doubly charged $\mathrm{Ar}$ atom $\left(\mathrm{NeAr}^{-2}\right)$.

Here, we discuss energy and charge transfer in addition to the subsequent fragmentation in nano-structured systems after site-selective ionization. We demonstrate that the fragmentation dynamics of heterogeneous clusters differ substantially depending on whether the ionization occurs on the surface or in the bulk of the cluster. Furthermore, the data give the first experimental hint that an ETMD-like process of enhanced charge transport takes place in extended, heterogeneous systems. The data were reproduced in two separate experiments.

The experiments were carried out during two-bunch operation at the beamlines 10.0.1 and 11.0.2 of the Advanced Light Source at the Lawrence Berkeley National Laboratory using the electron-ion coincidence setup described in Refs. [11,12]. An ion time-of-flight spectrometer (iTOF) [13] operated with pulsed extraction fields was combined with a high-resolution electron time-of-flight spectrometer (eTOF) [14] mounted at the magic angle of $54.7^{\circ}$ with respect to the polarization vector in the plane perpendicular to the light propagation direction and the axis of the iTOF. The heterogeneous core-shell clusters crossing the photons in the interaction region of the spectrometer were produced by supersonic expansion of a rare-gas mixture of $2 \% \mathrm{Xe}$ in $\mathrm{Ar}$ through a liquid-nitrogen cooled $(110 \mathrm{~K})$, conical nozzle $\left(D=100 \mu \mathrm{m}, \alpha=7.5^{\circ}\right)$ at 1.3 bar. The chosen mixing ratio gives rise to a $\mathrm{Xe}$ core with an ArXe interface surrounded by an Ar surface [15-17]. According to the scaling laws by Hagena [18], the average 


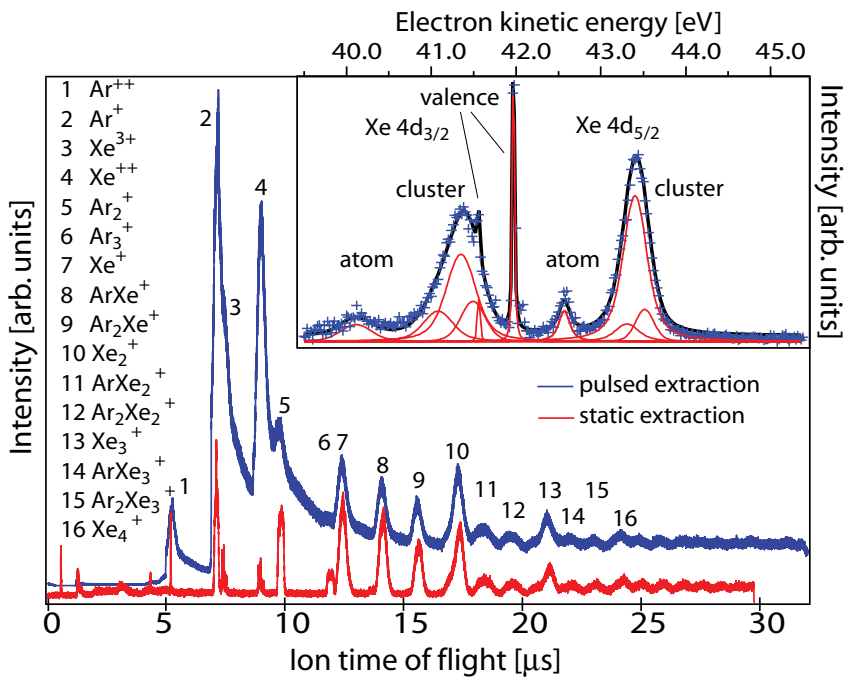

FIG. 1. (Color online) Ion time-of-flight spectra of ArXe cluster fragments after photoexcitation at $110 \mathrm{eV}$ [blue (dark gray) line] measured in coincidence with the angle-resolved detection of an electron compared to a measurement with static extraction fields [thin (light gray) red line]. The inset shows the corresponding $\mathrm{Xe} 4 d$ photoelectron spectrum. The two sharp lines that partially overlap with the $4 d_{3 / 2}$ cluster peak result from fast valence electrons produced by the subsequent photon bunch. The average cluster size $\langle N\rangle$ is smaller than 8000 .

size of pristine Ar clusters under these expansion conditions is $\langle N\rangle=8000$. For heterogeneous systems, these scaling laws give an upper estimate since the average cluster size is expected to be smaller due to different binding energies leading to an increased evaporation rate of the lighter compound [17].

An ion time-of-flight spectrum of ArXe cluster fragments extracted after angle-resolved detection of an electron is shown in Fig. 1. Also shown is a similar spectrum taken at the same photon energy and for identical expansion conditions but measured with static extraction voltages. In the latter, the mass resolution is much better since the lines are not broadened by the pulsed extraction. In addition it shows less contribution from residual gas like $\mathrm{Xe}^{2+}$ since a smaller skimmer was used for this measurement.

Two excitation energies, $110 \mathrm{eV}$ and $291.2 \mathrm{eV}$, were chosen for the measurement reported in this letter. These photon energies lie about $42 \mathrm{eV}$ above the $\mathrm{Xe} 4 d$ and $\operatorname{Ar} 2 p$ photoionization thresholds. The inset of Fig. 1 presents the kinetic energy spectrum of Xe $4 d$ electrons at $110 \mathrm{eV}$. Due to different numbers of neighboring atoms, the electron binding energies in clusters differ among bulk, interface and surface [16]. Therefore, a separation between cluster and residual gas and even among surface, interface, and bulk electrons becomes possible. When measuring the fragment ions in coincidence with the cluster photoelectrons, the dependence of the fragmentation on the site of the initial photoionization can thus be determined. In the following, we concentrate on coincidences with $\mathrm{Xe} 4 d_{5 / 2}$ and $\operatorname{Ar} 2 p_{3 / 2}$ photoelectrons since no valence or Auger lines are laying beneath these cluster peaks.

In order to trace the dependence of energy transfer and fragmentation dynamics to the ionization site, we set filters on the different cluster fragments and analyze the corresponding

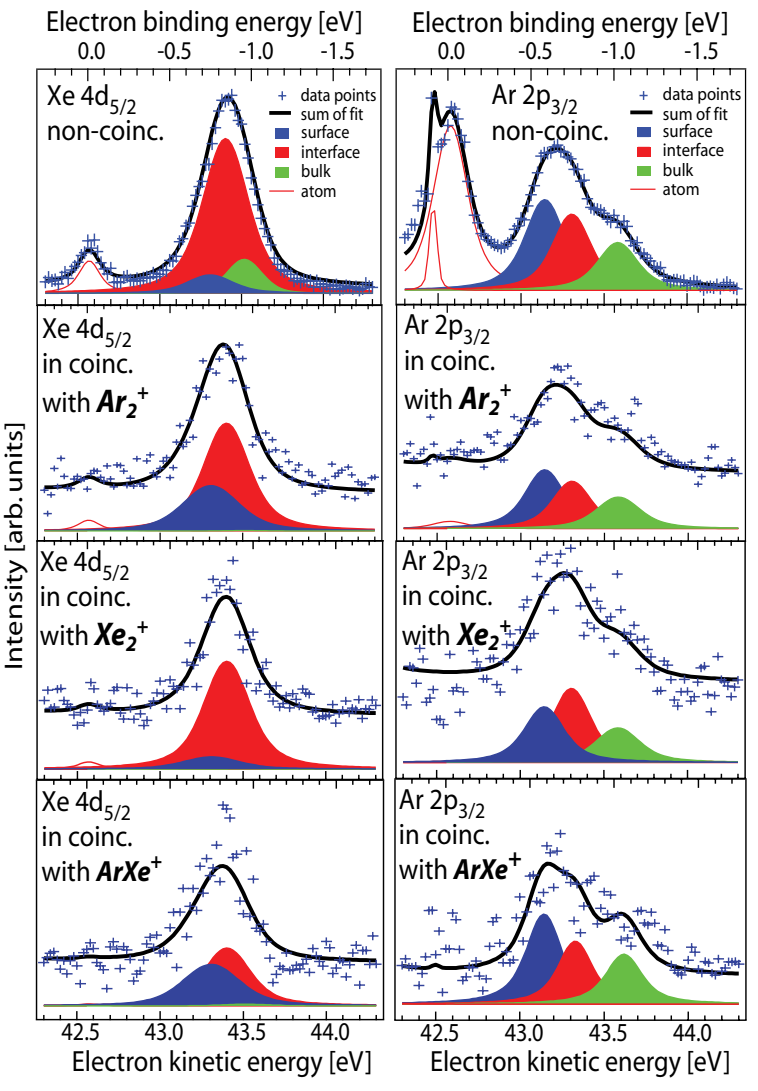

FIG. 2. (Color online) $\mathrm{Xe} 4 d_{5 / 2}$ (left) and Ar $2 p_{3 / 2}$ (right) photoelectron spectra without selection of ionic coincidences (top) and in coincidence with $\mathrm{Ar}_{2}^{+}, \mathrm{Xe}_{2}^{+}$, and $\mathrm{XeAr}^{+}$fragments (bottom panels).

coincident electron spectra with the least-squares curve fitting method. First, each cluster peak in the noncoincident electron spectra [Fig. 2 (top)] was fitted by three Voigt profiles for the surface, interface, and bulk contributions. The resulting Lorentzian and Gaussian peak widths and positions were then fixed and the same fit was applied to the coincidence spectra. We chose this way of curve fitting since it is a standard method for core shell system and is well accepted [16].

The left column in Fig. 2 depicts $\mathrm{Xe} 4 d_{5 / 2}$ photoelectron spectra in coincidence with three different cluster fragments after excitation at $110 \mathrm{eV}$. While pristine Xe clusters produced under similar gas expansion conditions show a clear separation between surface and bulk contribution [19], the spectra for mixed cluster are dominated by the interface signal. Therefore we assume a cluster structure dependence on the ionization and fragmentation process or a different cluster size distribution for mixed clusters.

The right-hand side shows $\operatorname{Ar} 2 p_{3 / 2}$ electron spectra in coincidence with the same fragments after excitation at $291.2 \mathrm{eV}$. The intensity ratios among surface, interface, and bulk peaks in these spectra vary strongly and one can immediately conclude that the production of these fragments strongly depends on the site of the ionization. The surprisingly high amount of $\mathrm{Ar}$ fragments measured in coincidence with electrons emerging from the bulk can be attributed to not perfectly separated $\mathrm{Ar}$ shell and Xe core as was deduced from previous works [16].

To obtain a more detailed and quantitative insight into energy and charge transfer processes as well as fragmentation 
TABLE I. Relative intensities of Xe $4 d_{5 / 2}$ and Ar $2 p_{3 / 2}$ surface (surf.), interface (interf.) and bulk electron peaks in coincidence with charged cluster fragments at $110 \mathrm{eV}$ (top) and $291.2 \mathrm{eV}$ (bottom) photon energy, respectively. Also shown are the relative intensities of surface, interface, and bulk in the "noncoincident" spectrum (all) and the contribution of the coincidences with each fragment to the total coincident electron signal. Listed are only fragments with $1 \%$ of the total cluster signal or more. The most intense coincidences are in bold.

\begin{tabular}{lllllllllllllll}
\hline \hline & & $\mathrm{All}$ & $\mathrm{Ar}^{2+}$ & $\mathrm{Ar}^{+}$ & $\mathrm{Ar}_{2}^{+}$ & $\mathrm{Ar}_{3}^{+}$ & $\mathrm{Xe}^{2+}$ & $\mathrm{Xe}^{+}$ & $\mathrm{Xe}_{2}^{+}$ & $\mathrm{Xe}_{3}^{+}$ & $\mathrm{ArXe}^{+}$ & $\mathrm{Ar}_{2} \mathrm{Xe}^{+}$ & $\mathrm{ArXe}_{2}^{+}$ & $\mathrm{Ar}_{2} \mathrm{Xe}_{2}^{+}$ \\
\hline $\mathrm{Xe} 4 d_{5 / 2}$ & Surface & 0.09 & 0.26 & $\mathbf{0 . 8 0}$ & 0.33 & $\mathbf{0 . 9 5}$ & 0.41 & 0.35 & 0.11 & 0.02 & 0.45 & $\mathbf{0 . 5 1}$ & 0.32 & $<0.01$ \\
$\mathrm{Xe} 4 d_{5 / 2}$ & Interface & $\mathbf{0 . 7 9}$ & $\mathbf{0 . 5 8}$ & 0.14 & $\mathbf{0 . 6 7}$ & 0.05 & $\mathbf{0 . 4 4}$ & $\mathbf{0 . 4 9}$ & $\mathbf{0 . 8 6}$ & $\mathbf{0 . 9 6}$ & $\mathbf{0 . 5 4}$ & 0.37 & $\mathbf{0 . 4 4}$ & $\mathbf{0 . 5 1}$ \\
$\mathrm{Xe} 4 d_{5 / 2}$ & Bulk & 0.12 & 0.16 & 0.05 & $<0.01$ & $<0.01$ & 0.15 & 0.16 & 0.03 & 0.02 & 0.01 & 0.12 & 0.24 & 0.48 \\
$\mathrm{Xe} 4 d_{5 / 2}$ & Total & 1.0 & 0.01 & $\mathbf{0 . 1 1}$ & $\mathbf{0 . 2 0}$ & 0.09 & 0.04 & $\mathbf{0 . 1 2}$ & $\mathbf{0 . 1 1}$ & 0.08 & 0.07 & 0.05 & 0.06 & 0.06 \\
$\mathrm{Ar} 2 p_{3 / 2}$ & Surface & $\mathbf{0 . 4 3}$ & 0.15 & $\mathbf{0 . 5 3}$ & $\mathbf{0 . 4 4}$ & $\mathbf{0 . 5 1}$ & 0.19 & $\mathbf{0 . 3 6}$ & 0.30 & $\mathbf{0 . 4 9}$ & $\mathbf{0 . 4 3}$ & $\mathbf{0 . 4 6}$ & $\mathbf{0 . 4 3}$ & $\mathbf{0 . 4 6}$ \\
$\mathrm{Ar} 2 p_{3 / 2}$ & Interface & 0.35 & $\mathbf{0 . 7 0}$ & 0.38 & 0.34 & 0.39 & $\mathbf{0 . 5 0}$ & 0.33 & $\mathbf{0 . 3 9}$ & 0.33 & 0.32 & 0.27 & 0.25 & 0.28 \\
$\mathrm{Ar} 2 p_{3 / 2}$ & Bulk & 0.22 & 0.15 & 0.09 & 0.22 & 0.10 & 0.31 & 0.31 & 0.31 & 0.18 & 0.25 & 0.27 & 0.32 & 0.27 \\
$\mathrm{Ar} 2 p_{3 / 2}$ & Total & 1.0 & 0.01 & $\mathbf{0 . 1 6}$ & $\mathbf{0 . 1 0}$ & $\mathbf{0 . 1 0}$ & 0.04 & $\mathbf{0 . 1 8}$ & $\mathbf{0 . 1 2}$ & 0.02 & $\mathbf{0 . 1 0}$ & 0.08 & 0.05 & 0.04 \\
\hline \hline
\end{tabular}

dynamics, we analyzed the relative contribution from the surface, interface and bulk to the photoelectron intensities measured in coincidence with each of the fragments. The results are summarized in Table I along with the corresponding relative intensities in the noncoincident electron spectrum as well as the contribution of the coincidences with each fragment to the total coincident electron signal.

The first striking result is the fact that after selective ionization of only $\mathrm{Xe}$ (or Ar) cluster atoms, all fragment species were detected: $\mathrm{Xe}_{n}^{+}, \mathrm{Ar}_{n}^{+}$, and $\mathrm{Ar}_{n} \mathrm{Xe}_{m}^{+}$fragments. Hence, charge or energy must have been transferred from the ionized atom to another site in the cluster. It is also noteworthy that in contrast to the prediction of the core-shell model, we measured a small but significant amount of surface contribution for the $\mathrm{Xe} 4 d$ photoelectrons as well as a bulk contribution of the Ar $2 p$ photoelectrons, suggesting that Ar and $\mathrm{Xe}$ are not as strongly segregated as reported previously [16]. Comparing the noncoincident and coincident Xe $4 d$ electron spectra (Fig. 2) as well as the values in Table I, it stands out that the bulk contribution is much more intense in the noncoincident spectra than in the sum of all the considered coincident spectra. We explain this by the fact that besides fragmentation into small fragments, clusters also fragment into very large singly or doubly charged fragments containing several hundred or thousand atoms, which have very long flight times and are detected with very poor efficiency due to their large mass and thus do not show up as recognizable peaks in the mass spectrum. These heavy fragments apparently originate mainly from the bulk and are hence responsible for the missing bulk intensity in the coincidence spectra. This discrepancy is a lot smaller for the ionization of $\operatorname{Ar} 2 p$, indicating that heavy fragments are produced less frequently in the case of surface ionization.

At $110 \mathrm{eV}$, according to Table I (top), $\mathrm{Ar}_{2}^{+}, \mathrm{Xe}_{2}^{+}$, and $\mathrm{Xe}^{+}$, $\mathrm{Ar}^{+}$are the most common (small) fragmentation products after ionization of the Xe atoms in the cluster bulk and interface. From ion yield measurements on atomic Xe, it is known that after photoionization at this photon energy, a second electron is released through Auger decay in $75 \%$ of all cases [20]. Since only a rather weak signal from doubly charged $\mathrm{Xe}^{2+}$ was observed in coincidence with the $4 d$ photoelectrons, it can be assumed that at least one positive charge is transferred to a direct neighbor. At the interface, the ionized $\mathrm{Xe}$ atom is surrounded by Xe as well as Ar atoms, thus charge transfer to both elements is possible. As the amount of Ar signal (41\%, sum of all pure Ar fragments) measured after Xe ionization exceeds the amount of Xe signal (35\%, sum of all pure $\mathrm{Xe}$ fragments), positive charge is transferred preferentially from Xe to Ar. This can be explained by the smaller interatomic separation between $\mathrm{Xe}^{+}$and $\mathrm{Ar}$ compared to $\mathrm{Xe}^{+}$and $\mathrm{Xe}$ [21], which leads to a larger overlap of the valence orbitals and therefore to higher charge transfer rate. Thus, charge migration between neighboring atoms of different elements or, in other words, from bulk to surface takes place. After electrons are transferred from Ar to Xe, it is most likely that the ion builds a dimer ion with a neighboring Ar atom (20\%) instead of a $(\mathrm{ArXe})^{+}$dimer $(7 \%)$ since the $\mathrm{Ar}_{2}^{+}$binding energy $(1.19 \mathrm{eV})$ exceeds the binding energy with $\mathrm{Xe}\left(\mathrm{Xe}_{2}^{+}, 0.79 \mathrm{eV}\right)$ [21]. After interface ionization, the positive charge is apparently hopping between neighboring atoms until it localizes on the energetically most favored site. The formation of pure Xe fragments or mixed fragments also occurs but less frequently (Table I, top). In agreement to results on pristine clusters [22], the charge localization seems to depend on the ionization environment.

One coincidence channel of particular interest is $\mathrm{Ar}_{3}^{+}$with a total yield of $9 \%$, which is almost exclusively produced after $\mathrm{Xe} 4 d$ ionization at the surface (95\%). It is the largest fragment occurring in a significant amount that contains none of the initially ionized Xe atoms. Here, after Xe $4 d$ ionization positive charge had to again be transferred to a neighboring $\mathrm{Ar}$ atom, which subsequently formed an Ar trimer. Subsequently it seems energetically allowed to build an Ar trimer. Most likely, the Xe atom on the surface is surrounded only by $\mathrm{Ar}$ atoms.

Compared to the results obtained for the Xe $4 d$ cluster ionization at $110 \mathrm{eV}, \operatorname{Ar} 2 p$ ionization of the cluster at a photon energy of $291.2 \mathrm{eV}$, which predominantly ionizes Ar atoms that are located in the outer shell of the cluster, leads to significantly different coincidence channels (Table I, bottom). Based on our results and the theoretical predictions, it can be assumed that after the ionization of Ar atoms, energy is transferred through the ICD process between Ar-Ar or Ar-Xe as described above, or through direct charge transfer. Since energy transfer processes such as ICD take longer for surface than for bulk atoms, the fragmentation dynamics differ depending on the site of the initial ionization, as can be seen in Table I. Ions on the surface have less neighbors compared to bulk atoms thus 
less collision partners. Driven by Coulomb repulsion, they will leave the cluster faster than bulk ions. After surface ionization, the cluster therefore breaks up before dominant decay channels can develop, and the size distribution of possible fragments becomes broader (Table I, bottom). After interface and bulk ionization, fragmentation takes longer because more atoms need to be rearranged before the ions can escape. During the time of bulk rearrangement, a quasiequilibrium for the charge distribution sets in and distinct decay channels can be initialized. Besides the coincidences stemming from surface ionization, which dominate the total signal at 291.2 $\mathrm{eV}$, there are three fragments that are mostly produced after interface ionization: $\mathrm{Ar}^{2+}$ (1\% of the total ion yield), $\mathrm{Xe}^{2+}(4 \%)$, and $\mathrm{Xe}_{2}^{+}(12 \%)$. For $\mathrm{Xe}^{2+}$, a very efficient charge transfer between neighboring $\mathrm{Ar}$ and $\mathrm{Xe}$ atoms has to be considered, which we attribute to ETMD. For ETMD to occur, the double ionization threshold (DP) of a neighboring $\mathrm{Xe}$ atom needs to be energetically lower than the inner valence ionization potential (IP) of Ar. This is not the case for isolated atoms (IP $\mathrm{Ar} \mathrm{3s}$ : $29.3 \mathrm{eV}$, DP Xe $5 p$ : $33.1 \mathrm{eV}$ ). For cluster bulk atoms however, the ionization potential is lowered due to polarization forces and it could therefore become energetically possible to fulfill this conditions necessary for ETMD. It should be emphasized that this transfer process is possible only in heterogeneous systems. It remains unclear how a doubly charged fragment can leave the cluster without further charge transfer, which also stands in strong contrast to results we gained on pristine clusters, where no doubly charged fragments were observed in coincidences with photoelectrons emitted from the cluster [23].

In conclusion, we elucidated charge and energy transfer and subsequent fragmentation dynamics in ArXe core shell systems after site-selective photoionization using a highresolution electron-ion coincidence technique. Surprisingly, coincidences with Ar $2 p$ electrons measured after cluster surface ionization do not show as distinct fragmentation channels as coincidences with $\mathrm{Xe} 4 d$ electrons measured after cluster interface and bulk ionization. The latter clearly shows preferred decay channels. We attribute this strong dependence of the fragmentation scenario of a nanosystem such as ArXe clusters on the site of the initial ionization (surface, interface, bulk) to the influence of the specific environment of the ionized cluster atom on the decay and charge localization times. After surface ionization, the system fragments very fast in a broad range of decay channels. Fragmentation, however, takes longer after bulk ionization due to inner rearrangement favoring a small number of energetically dominant decay channels. In addition, strong hints for a very effective charge transfer from ionized $\mathrm{Ar}$ atoms to surrounding $\mathrm{Xe}$ atoms were measured, which we attribute to the electron transfer mediated decay (ETMD) mechanism.

This work was supported by the Office of Basic Energy Sciences, US Department of Energy, Chemical Sciences, Geosciences and Biosciences Division. M.H. and D.R. are grateful to the Alexander von Humboldt Foundation for support through the Feodor Lynen Program.
[1] P. Cheng, J. Spencer Baskin, A. Zewail et al., Proc. Natl. Acad. Sci. USA 103, 10570 (2006).

[2] R. Baughman, A. Zakhidov, W. de Herr et al., Science 297, 787 (2002).

[3] T. Jahnke, A. Czach, M. Schöffler, S. Schössler, A. Knapp, M. Käsz, J. Titze, C. Wimmer, K. Kreidi, R. Grisenti et al., Phys. Rev. Lett. 93, 163401 (2004).

[4] T. Osipov, C. L. Cocke, M. H. Prior, A. Landers, T. Weber, O. Jagutzki, L. Schmidt, H. Schmidt-Böcking, R. Dörner et al., Phys. Rev. Lett. 90, 233002 (2003).

[5] M. Schöffler, J. Titze, N. Petridis, T. Jahnke, K. Cole, L. Schmidt, A. Czasch, D. Akoury, O. Jagutzki, J. Williams et al., Science 320, 920 (2008).

[6] Y. Morishita, X.-J. Liu, N. Saito, T. Lischke, M. Kato, G. Prümper, M. Oura, H. Yamaoka, Y. Tamenori, I. H. Suzuki et al., Phys. Rev. Lett. 96, 243402 (2006).

[7] Y. Morishita, N. Saito, I. H. Suzuki, H. Fukuzawa, X.-J. Liu, K. Sakai, G. Prümper, K. Ueda, H. Iwayama, K. Nagaya et al., J. Phys. B 41, 025101 (2008).

[8] V. Averbukh, I. B Müller, L. S. Cederbaum, Phys. Rev. Lett. 93, 263002 (2004).

[9] G. Öhrwall, M. Tchaplyguine, M. Lundwall, R. Feifel, H. Bergersen, T. Rander, A. Lindblad, J. Schulz, S. Peredkov, S. Barth et al., Phys. Rev. Lett. 93, 173401 (2004).

[10] J. Zobeley, R. Santra, L. Cederbaum et al., J. Chem. Phys. 115, 5076 (2001).

[11] D. Rolles, G. Pruemper, H. Fukuzawa, X.-J. Liu, Z. Pesic, R. Fink, A. Grum-Grzhimailo, I. Dumitriu, N. Berrah, K. Ueda et al., Phys. Rev. Lett. 101, 263002 (2008).
[12] G. Pruemper, D. Rolles, H. Fukuzawa, X.-J. Liu, Z. Pesic, I. Dumitriu, R. Lucchese, N. Berrah, K. Ueda et al., J. Phys. B 41, 215101 (2008).

[13] D. Rolles, Z. Pesic, M. Perri, R. Bilodeau, G. Ackermann, B. Rude, A. Kilcoine, J. Bozek, N. Berrah et al., Nucl. Instrum. Methods 261, 170 (2007).

[14] N. Berrah, B. Langer, A. A. Wills, E. Kukk, J. D. Bozek, A. Farhat, T. W. Gorczyca, J. Electron Spectrosc. Relat. Phenom. 103, 1 (1999).

[15] O. Danylchenko, Y. Doronin, S. Kovalenko, V. Samovarov et al., JETP Lett. 84, 324 (2006).

[16] M. Tchaplyguine, M. Lundwall, M. Gisselbrecht, G. Öhrwall, R. Feifel, S. Sorensen, S. Svensson, M. N., O. Bjørneholm et al., Phys. Rev. A 69, 031201(R) (2004).

[17] M. Hoener, C. Bostedt, H. Thomas, L. Landt, E. Eremina, R. Treusch, H. Wabnitz, T. Möller et al., J. Phys. B 41, 181001 (2008).

[18] O. Hagena, W. Obert et al., J. Chem. Phys. 56, 1793 (1972).

[19] D. Rolles, H. Zhang, Z. Pesic, R. Bilodeau, A. Wills, E. Kukk, B. Rude, G. Ackermann, J. Bozek, R. Diez Muino et al., Phys. Rev. A 75, 031201 (R) (2007).

[20] I. Suzuki N. Saito et al., J. Elec. Spec. Rel. Phen. 129, 71 (2003).

[21] W. Wadt et al., J. Chem. Phys. 68, 402 (1978).

[22] M. Hoener, C. Bostedt, S. Schorb, H. Thomas, L. Foucar, O. Jagutzki, R. Dörner, T. Möller et al., Phys. Rev. A 78, 021201(R) (2008).

[23] D. Rolles, M. Hoener, and N. Berrah (to be published, 2010). 